Bristol-Myers Squibb, Celgene, Crescendo, Eli Lilly, GSK, Janssen, Merck, Novartis, Pfizer Inc, Roche, UCB and Vertex, Jose Luis Rivas Shareholder of: Pfizer Inc, Employee of: Pfizer Inc, Salim Benkhalifa Shareholder of: Pfizer SAS, Employee of: Pfizer SAS, Palle Dahl Shareholder of: Pfizer Inc, Employee of: Pfizer Inc, Harry Shi Shareholder of: Pfizer Inc, Employee of: Pfizer Inc, Lisy Wang Shareholder of: Pfizer Inc, Employee of: Pfizer Inc

DOI: 10.1136/annrheumdis-2019-eular.417

\section{AB0418 INTERSTITIAL LUNG DISEASE IN PATIENTS WITH RHEUMATOID ARTHRITIS TREATED WITH TOFACITINIB}

Marisel Bejarano ${ }^{1}$, Maria Natalia Tamborenea ${ }^{2}$, Mario Alberto Goñi ${ }^{3}$, Lina Saldarriaga $^{4}$, Cecilia Pisoni $^{5}$, Rodrigo Garcia Salinas ${ }^{6}$, Mariana Salcedo $^{7}$, Claudia Helling ${ }^{8}$, Adrian Salas ${ }^{8} .{ }^{1}$ Hospital General de Agudos Bernardino Rivadavia, Rheumatology Unit, CABA, Argentina; ${ }^{2}$ Hospital General de Agudos Bernardino Rivadavia, CABA, Argentina; ${ }^{3}$ Instituto de Lucha Antipoliomielítica y Rehabilitación del Lisiado, Rosario, Argentina; ${ }^{4}$ Hospital Universitario San Jorge, Pereira, Colombia; ${ }^{5}$ CEMIC, caba, Argentina; ${ }^{6} \mathrm{Hospital}$ Italiano De La Plata, La Plata, Argentina; ${ }^{7}$ INSTITUTO DE INVESTIGACIONES CLINICAS DE ZÁRATE, Zarate, Argentina; ${ }^{8}$ Pfizer SRL, CABA, Argentina

Background: The presence of interstitial lung disease (ILD) is a common extra-articular manifestation in patients diagnosed with Rheumatoid Arthritis (RA), dyspnea being its cardinal symptom. Several of the drugs commonly used to treat this condition, such as TNF inhibitors (anti-TNF) and Disease Modifying Antirheumatic Drugs (DMARDs), have been implicated in the development or exacerbation of ILD. In regard to Tofacitinib, results of post hoc analysis of its pivotal and extension studies report a numerically lower ILD incidence rate in the group that received this drug compared with placebo.

Objectives: To describe the evolution of dyspnea, spirometric parameters, DLCO and high resolution computed tomography (HRCT) findings in patients with RA diagnosis with associated PID who received Tofacitinib for a period of 12 months.

Methods: Patients with a diagnosis of RA who fulfilled criteria ACR 1987/ ACR-EULAR 2010, with interstitial lung disease previously diagnosed from clinical findings, spirometry and/or HRCT were included. Tofacitinib was used in dose of $10 \mathrm{mg} /$ day orally. Design: Descriptive, retrospective, multicentric study. The following variables were analyzed: change at 12 months from baseline in the degree of dyspnea (according to the modified scale of dyspnea MMRC), forced vital capacity (FVC), DLCO and findings in HRCT. Continuous variables were reported as mean and standard deviation or median and interquartile range as appropriate. The categorical variables were reported as percentages.

Results: Fifteen patients were included. $60 \%$ (n: 9) were women. The mean age was 64.4 years $( \pm 10.92)$. The median time of evolution of RA was 9 years (IQR: 4-40). The median time of evolution of interstitial lung disease was 4 years (IQR: 1-24).13\% (n: 2) of the patients were receiving methotrexate (MTX) at the time of evaluation, $60 \%$ (n: 9) of the patients had previously received it. $80 \%(n: 12)$ had previously received DMARD different from MTX. 33\% of the patients ( $n$ : 5 ) had received previous biological treatment. All patients received tofacitinib at a dose of $10 \mathrm{mg} / \mathrm{day}$. $47 \%$ of patients $(n=7)$ received tofacitinib monotherapy and $53 \%(n=8)$ received it combined with DMARDsc. $27 \%$ (n: 4) of the patients had grade 3-4 dyspnea at baseline.Improvement in the dyspnea scale was observed in 8 patients, while in the rest, it remained stable. The forced vital capacity (FVC) at baseline was $<80 \%$ in 5 patients and $>80 \%$ in 2 patients. At 12 months, 4 patients achieved a FVC> $80 \%$. The average of DLCO at baseline was 45.6 ( \pm 18.84), with an improvement of $30 \%$ at 12 months in 4 patients. No progression of the disease was observed in the HRCT at 12 months in any of the patients evaluated.

Conclusion: The present preliminary study was performed with patients of daily practice, not being available in all cases, the corresponding respiratory functional examinations. However, despite these limitations, none of the patients showed worsening of dyspnea, with improvement in some patients. Regarding respiratory functional examinations and DLCO, not only they remained stable after treatment in the majority of patients evaluated, but 4 patients presented improvement with respect to baseline parameters. It is necessary to perform more studies, with systematized controls of the pulmonary function and images, to corroborate this hypothesis.

Disclosure of Interests: Marisel Bejarano: None declared, Maria Natalia Tamborenea: None declared, Mario Alberto Goñi: None declared, Lina Saldarriaga: None declared, Cecilia Pisoni: None declared, Rodrigo Garcia Salinas: None declared, Mariana Salcedo: None declared, Claudia Helling Employee of: I am an employee of Pfizer, Adrian Salas Consultant for: Pfizer, Employee of: I am an employee of Pfizer

DOI: 10.1136/annrheumdis-2019-eular.4896

\section{AB0419}

FRACTURE RISK IN PATIENTS ON LONG TERM GLUCOCORTICOIDS WITH INFLAMMATORY ARTHRITIS

Jemma Buchalter, Ahmed Elmahdi, Aine Gorman, Qutab Shah, Angela Camon, Ausaf Mohammad, Killian O'rourke. Midland regional hospital Tullamore, Rheumatology, Tullamore, Ireland

Background: A main stay treatment of inflammatory arthritis is glucocorticoids $(\mathrm{GCs})^{[1]}$. However, long term use of GCs has been associated with an increased risk of fracture, with studies estimating up to $12 \%$ of patients having sustained a fracture whilst on GC treatment ${ }^{[1][2]}$.

Objectives: The aim of this study was to determine the prevalence of fractures in a cohort of patients on long term GCs with a diagnosis of inflammatory arthritis.

Methods: A database of patients with inflammatory arthritis attending the rheumatology department at the Midlands Regional Hospital Tullamore, since 2009, was reviewed. Patients on long term GCs were identified and outpatient summaries were analysed. Data collection included age, sex diagnosis, vertebral fracture, other fracture sites and osteoporosis treatment. Results: As of September 2018, out of the 2118 patients with a diagnosis of inflammatory arthritis 364 were on long term GCs. $10 \%$ of patients had reported vertebral fractures on imaging. $36.4 \%$ of patients had other types of fractures reported on imaging; $20 \%$, and the most common site of fracture, were wrist fractures. $83.7 \%$ of those who suffered from a vertebral fracture were female. $18.2 \%$ of those who sustained fractures were not on any osteoporosis treatment.

Conclusion: The incidence of fracture in our cohort of patients was higher than suggested in previous studies. Furthermore, it highlights the need for patients on long term GCs to be given osteoporosis prevention therapy.

\section{REFERENCES}

[1] Buckley L, Guyatt G, Fink HA, Cannon M, Grossman J, Hansen KE, et al 2017 American College of Rheumatology Guideline for the Prevention and Treatment of Glucocorticoid-Induced Osteoporosis. Arthritis Rheumatol. 2017;69:1521-37

[2] Dam Kim, Soo-Kyung Cho, Byeongju Park, Eun Jin Jang, Sang-Cheol Bae and Yoon-Kyoung Sung. The Journal of Rheumatology March 2018, jrheum.170054

Disclosure of Interests: None declared

DOI: 10.1136/annrheumdis-2019-eular.8014

\begin{tabular}{l|l}
\hline AB0420 & A PROSPECTIVE, OBSERVATIONAL STUDY ON THE \\
CLINICAL EFFICACY AND SAFETY OF LEFLUNOMIDE \\
IN EGYPTIAN PATIENTS WITH ACTIVE RHEUMATOID \\
ARTHRITIS: CLEAR STUDY
\end{tabular}

Adel Elsayed. Ain Shams University, Department of Rheumatology, Cairo, Egypt

Background: Due to its anti-inflammatory and immunomodulatory properties, leflunomide can be used as a monotherapy or in combination with other conventional synthetic DMARDs (csDMARDs) in treating active RA. ${ }^{1}$ Objectives: The aim of this observational study was to assess efficacy and safety of leflunomide in Egyptian patients with active RA, whether used as first-line and/or add-on therapy to other csDMARDs, with or without steroids. Methods: Adult Egyptian patients with active RA, fulfilling the ACR/EULAR 2010 classification criteria, for whom leflunomide was prescribed, were included and followed up for 9 months. Leflunomide was prescribed at the physicians' sole discretion for patients who have previously experienced resistance, inadequate response or intolerance to other csDMARDs Data about CDAI score and $H A Q-D I$ score were acquired from patients who fulfilled the eligibility criteria, along with patients' demographics, medical history, physical examination, concomitant medications, and RA history including disease duration, previous RA medications and treatment response. This study was registered on clinicaltrials.gov (NCT03599986) and the preliminary results were previously published in $2018 .^{2}$

Results: A total of 398 patients were enrolled in this study. Three hun dred sixty-six patients with a mean age of 43.7 (SD 10.2) years and median disease duration of 2 (IQR 5.5) years were eligible for efficacy evaluation; 309 (84.4\%) were female. We found that $86.1 \%$ of patients were leflunomide naïve. Patients who previously received leflunomide $(13.9 \%)$ had a wash out period of at least 6 months prior to study enrolment. Patients were either prescribed leflunomide $20 \mathrm{mg} / \mathrm{day}(37.7 \%)$ or were prescribed a loading dose of $100 \mathrm{mg} /$ day for the first 3 days of treatment then maintained with $20 \mathrm{mg} /$ day $(62.3 \%)$. Leflunomide was prescribed as first-line therapy in $21.1 \%$ of patients, as add-on therapy to other csDMARDs without steroids in $34 \%$, and as add-on therapy to other csDMARDs with steroids in $44.9 \%$. The mean total CDAI score 
was significantly decreased $(p<0.001)$ from 36.8 (14.9) at baseline, to reach 12.5 (10.3) after 9 months of treatment, with a reduction of $64 \%$. In a similar pattern, the mean HAQ-DI score was significantly decreased $(p<0.001)$ from $1.5(0.7)$ at baseline to reach $0.6(0.6)$ after 9 months of treatment, with a reduction of $57 \%$. Figures 1 and 2 show changes in both CDAl and HAQ-DI scores throughout the study.

Figure 1: CDAI Score at Baseline, Month 3 and Month 9

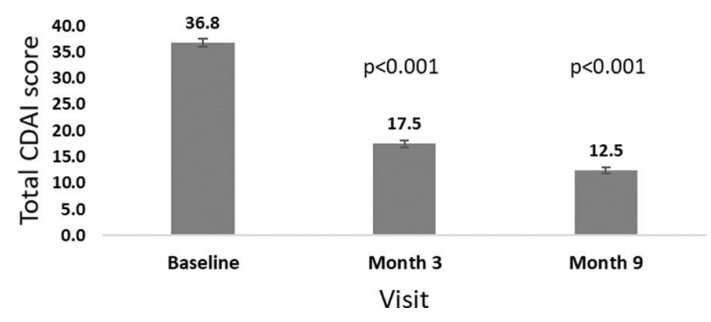

Figure 2: HAQ-DI at Baseline, Month 3 and Month 9

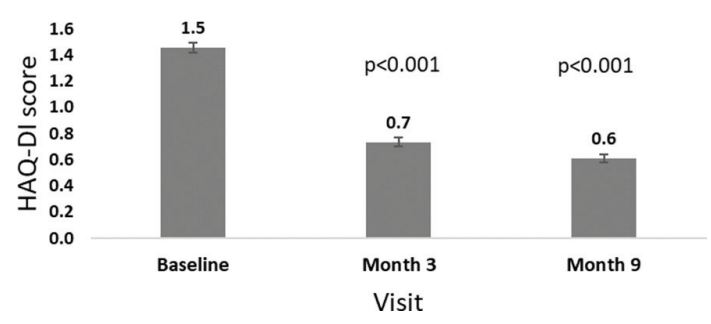

Figure 3: Disease severity at Baseline, Month 3 and Month 9

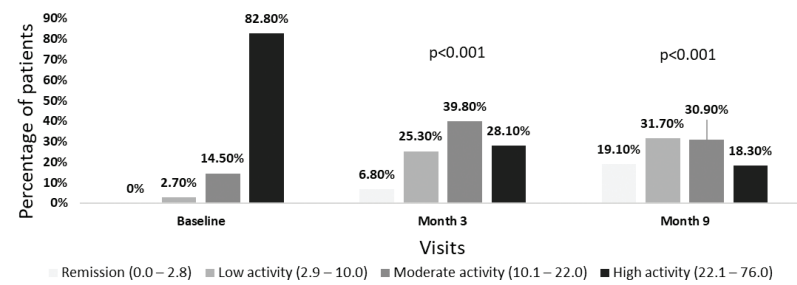

Abstract Figure 3. shows that disease severity was significantly improved $(p<0.001)$ throughout the study, and $19.1 \%$ of patients were at remission after 9 months of treatment.

Twenty-three adverse events (AEs) were reported in 20 patients (5\%) throughout the study period; most frequently, increased hepatic enzymes (6 cases, $1.5 \%$ ), increased swollen/tender joint count (2 cases, $0.5 \%$ ), decreased white blood cell count (2 cases, $0.5 \%$ ), and abdominal discomfort (2 cases, $0.5 \%$ ). None of the reported AEs were related to treatment with leflunomide. Conclusion: Results from this study demonstrate that leflunomide may offer an effective, tolerable and safe treatment -whether used as a monotherapy or in combination with other csDMARDs- in Egyptian patients with active RA.

\section{REFERENCES}

[1] Alldred A, Emery P. Leflunomide: a novel DMARD for the treatment of rheumatoid arthritis. Expert opinion on pharmacotherapy. 2001;2(1):125-137.

[2] El Sayed A, Clinical efficacy and safety of leflunomide in Egyptian patients with active rheumatoid arthritis: CLEAR interim results. The open rheumatology journal. 2018;12(1):323-331.

Acknowledgement: Thanks to all Investigators: A Abd El Hamid, A Darwish, AE Hilal, A Elgerby, AF Abou Gamal, A Labib, AS Omar, D Abd El Mohsen, D Fayez, E Abda, F Elshabacy, M El Shehaly, M El Tayel, M El Wakd, M Mortada, N Mohannad, R Abd El Kader, S El Bakry, S Refaat, and S Zaki. Data management, statistical analysis, site monitoring activities, and editorial support were provided by DATACLin CRO and funded by EVAPHARMA.

Disclosure of Interests: Adel Elsayed Grant/research support from: This study was funded by EVAPHARMA.

DOI: 10.1136/annrheumdis-2019-eular.4595

\section{$\mathrm{AB} 0421$ \\ METHOTREXATE COMPLIANCE AMONG PATIENTS WITH CHRONIC INFLAMMATORY DISEASE OR CONNECTIVE TISSUE DISEASE}

Elisabeth FILHOL ${ }^{1}$, Charlotte Hua ${ }^{1}$, Francoise Flaisler ${ }^{2}$, Cédric Lukas ${ }^{3}$, Bernard Combe $^{3}$, Jacques Morel ${ }^{3}$, Cecile Gaujoux-Viala'. ${ }^{1}$ Nîmes University Hospital, Montpellier University, Nîmes, France; ${ }^{2}$ Nîmes University Hospital, Nîmes, France; ${ }^{3}$ Lapeyronie hospital, Montpellier University, Montpellier, France

Background: Methotrexate (MTX) is widely used in rheumatology. About $30 \%$ of patients receiving MTX do not respond. One of the explanations could be poor adherence.

Objectives: The purpose of this study was to assess adherence and to identify predictive factors influencing it.

Methods: 188 patients with MTX for at least 3 months (rheumatoid arthritis (RA), peripheral spondyloarthritis (PS), psoriatic arthritis (PA), lupus, Sjögren's Syndrome (SS)) were enrolled between May 2017 and May 2018. Each patient completed a questionnaire including socio-demographic data, disease and MTX characteristics, 4-question Morisky, rheumatism activity scores including DAS 28, quality of life (EQ-5D), fibromyalgia (FiRST), anxiety and depression (HADS), catastrophism (PCS-CF), coping (WCC-R) and whether or not to participate in a therapeutic education program.

Results: 188 were recruited, of which $75 \%$ were women, with a mean age of $61.4+/-13.24$ years: RA $(77.12 \%)$, PS $(7.98 \%)$, PA $(10.11 \%)$, lupus $(2.66 \%)$ and SS (2.13\%). $68.6 \%$ were observant, versus $31.4 \%$ unobservant minor $(28.2 \%)$ or major $(3.2 \%)$ patients. In multivariate analysis, two models were performed and found a poor adherence in case of depression OR: 3.120 [1.051; 9.354], catastrophism OR: 3.974 [1.119; 14.108], anti-CCP antibodies OR: 4.019 [1.277; 12.653] in RA patients and depression OR: 2.715 [1.237; 5.963], absence of comorbidities OR: $2.309[1.085 ; 4.915]$ with all rheumatism.

Conclusion: Some modifiable factors such as depression and catastrophism were associated with the risk of non-adherence with MTX. It is important to consider depression and catastrophism when taking care of our patients to improve adherence and avoid therapeutic escalations.

Disclosure of Interests: Elisabeth FILHOL: None declared, Charlotte Hua: None declared, Francoise Flaisler: None declared, Cédric Lukas: None declared, Bernard Combe Consultant for: Abbvie, Bristol-Myers Squibb, Gilead, Janssen, Eli Lilly, MSD, Novartis, Pfizer, Roche-Chugai, Sanofi, UCB, Jacques Morel: None declared, Cecile Gaujoux-Viala Consultant for: Speaking and/or consulting fees from AbbVie, Amgen, Bristol-Myers Squibb, Celgene, Eli Lilly, Gilead, Janssen, Merck-Serono, Medac, Nordic Pharma, Novartis, Pfizer, Roche, Sandoz, Sanofi and UCB Pharma. Speakers bureau: Speaking and/or consulting fees from AbbVie, Amgen, Bristol-Myers Squibb, Celgene, Eli Lilly, Gilead, Janssen, Merck-Serono, Medac, Nordic Pharma, Novartis, Pfizer, Roche, Sandoz, Sanofi and UCB Pharma.

DOI: 10.1136/annrheumdis-2019-eular.4682

\section{$\mathrm{AB} 0422$ \\ ACHIEVEMENT OF PATIENT ACCEPTABLE SYMPTOM STATE (PASS) IN RHEUMATOID ARTHRITIS PATIENTS TREATED WITH BARICITINIB}

Cristina Garufi, Francesca Spinelli, Fulvia Ceccarelli, Ilaria Duca, Silvia Mancuso, Cristiano Alessandri, Guido Valesini, Fabrizio Conti. Policlinico Umberto I, Rheumatology, Department of Internal Medicine and Medical Specialities, Sapienza University of Rome, Roma, Italy, Roma, Italy

Background: Baricitinib, a newly approved drug for the treatment of patients with moderate to severe rheumatoid arthritis (RA), is a selective inhibitor of Janus kinase 1 and 2. In a patient-centered care, patientreported outcomes (PROs) are really helpful to better evaluate the disease activity and the response to therapy. Between them, Patient Acceptable Symptom State (PASS) is defined as the highest level of symptoms beyond which patients consider themselves well [1], and consists of a single question with dichotomized answer (yes or no): "Considering all the different ways your disease is affecting you, if you would stay in this state for the next months, do you consider that your current state is satisfactory?". In randomized controlled trials baricitinib showed significant improvement of pain, fatigue, tiredness and assessment of disease [2].

Objectives: The aim of the present study was to investigate the effect of baricitinib on PASS one month from the beginning of therapy. Further aim was to quantify the time patients needed to reach an acceptable state of health and determine the clinical variables associated with PASS. Methods: Patients affected by RA according to 2010 ACR criteria, starting treatment with baricitinib as clinically indicated, were consecutively enrolled; PASS was determined after 1 month of treatment (T1); days to 